\title{
The terminal care of patients with lung cancer
}

\author{
R. G. TWYCROSS \\ M.A., B.M., M.R.C.P. \\ St Christopher's Hospice, Lawrie Park Road, London SE26 6DZ
}

\begin{abstract}
Summary
Lung cancer is the commonest form of malignant disease seen at St Christopher's Hospice. More than $35 \%$ of the male and about $8 \%$ of the female cancer patients are admitted with this diagnosis. This means that each year approximately 100 patients with lung cancer are amitted and cared for at the hospice. The more common symptoms experienced by 185 consecutive terminal lung cancer patients admitted to St Christopher's Hospice are listed in Table 1.
\end{abstract}

\section{Weakness}

When admitted, 133 patients $(72 \%)$ complained of weakness. Of these, seven were hemiplegic, three paraplegic and one had polyneuritis: the rest, however, just felt weak. In some the weakness was so severe that they became bedfast, precipitating admission to hospital.

TABle 1. Common symptoms in 185 patients admitted to $\mathrm{St}$ Christopher's Hospice in 1970 and 1971 with advanced lung cancer

\begin{tabular}{ll}
\hline 1. Weakness & $72 \%$ \\
2. Pain & $63 \%$ \\
3. Dyspnoea & $61 \%$ \\
4. Anorexia & $59 \%$ \\
5. Weight loss & $59 \%$ \\
6. Cough & $58 \%$ \\
7. Nausea/vomiting & $28 \%$ \\
8. Haemoptysis & $24 \%$ \\
\hline
\end{tabular}

\section{Case history 1}

Mr C.J.B., a retired railway porter, was 68 when he died in May 1972. In 1969, he had a right lower lobectomy following the discovery of lung cancer. In September 1970 a local recurrence was noted. Since the beginning of 1972 he had become increasingly unwell He began to abuse his relatives, withdrew from social contacts and repeatedly threatened suicide. When admitted in April 1972, he was dirty, unkempt and for the previous six weeks had been virtually bedfast due to marked weakness. He was anorexic and constipated.
Initial treatment comprised prednisone $5 \mathrm{mg}$ t.d.s. and amitriptyline $25 \mathrm{mg}$ daily. He spent the whole of the next day in the dayroom and ate a light diet. After a week the dose of amitriptyline was increased to $50 \mathrm{mg}$ daily. He steadily improved. After 2 weeks he was fully mobile, no longer depressed and eating normally. He required a course of antibiotics for a troublesome cough productive of green sputum. After 4 weeks he suddenly became confused and died less than an hour later.

Our experience suggests that prednisone should be prescribed in all cases of moderate or severe generalized weakness associated with terminal lung cancer. The usual dose is $5 \mathrm{mg}$ t.d.s. More often than not it helps and sometimes the effect is dramatic, as in the above case. It is probable that, in the majority of cases, the weakness is caused by hypercalcaemia induced by the malignant process. An elevated plasma ionized calcium concentration in cancer can lead to a wide variety of symptoms, including pain of a diffuse nature, nausea and vomiting, generalized weakness, tiredness, lassitude, drowsiness and other mental symptoms. $15-20 \%$ of all cancer patients develop hypercalcaemia at some stage of their illness and in breast and lung cancer the percentage is even higher than this overall figure (Watson, 1966). Unfortunately, in the presence of hypoalbuminaemia the total plasma calcium concentration may remain normal despite a definite elevation in the ionized calcium level. Consequently, it is necessary to act on a high level of clinical suspicion rather than rely on biochemical estimation. Both cortisone acetate and prednisone will correct the majority of hypercalcaemic states associated with malignant disease.

Rightly or wrongly we do not screen patients routinely for biochemical abnormalities. Perhaps we ought, as we should then be able to exclude patients in whom glucocorticosteroid therapy might make matters worse. Recently, a 72-year-old man with lung cancer was admitted with severe, incapacitating weakness. On account of previously diagnosed hypopituitarism he had been receiving cortisone acetate $50 \mathrm{mg}$ daily for 5 years. His serum potassium concentration was $2.4 \mathrm{mEq} / \mathrm{l}$. He improved when 
prescribed effervescent potassium chloride $1 \mathrm{~g}$ t.d.s. and after 10 days his serum potassium had risen to $3.7 \mathrm{mEq} / \mathrm{l}$. His improvement, however, was shortlived and after this he began to deteriorate and died a few days later.

Pain

Pain experienced by terminal cancer patients may be due to a variety of causes (Table 2). Pain due to

\begin{tabular}{l}
$\begin{array}{l}\text { TABLE 2. Miscellaneous causes of pain } \\
\text { in advanced malignant disease }\end{array}$ \\
\hline 1. Fear, anxiety, depression \\
2. Constipation, haemorrhoids \\
3. Peptic ulcer \\
4. Musculoskeletal pains \\
5. Bedsores \\
6. Infection \\
7. Venous thrombosis \\
8. Pulmonary embolism
\end{tabular}

the malignant process itself is a chronic pain, usually constant even if variable in intensity. If one is to be successful in relieving such pain it is of fundamental importance to appreciate that acute and chronic pain are as different from one another as acute and chronic renal failure. It is all too easy for a physician to forget this when he probably has no personal experience of chronic pain. Unfortunately for our patients, a doctor's understanding of pain is usually taken from his own experience of acute pain-the toothache, the headache, the cut or bruise. As we are taught from childhood to regard this as a good and useful warning, it causes defensive reflexes and usually passes quite quickly. Chronic pain, whether malignant or not, does not and cannot fit into such a description. It is impossible to predict when it will end. It may well go on getting worse. It appears to be utterly meaningless and rapidly expands to occupy the entire life field. When that happens, life is no longer worth living.

Chronic pain consists of three components:
1. Physical
2. Mental
3. Social.

Much mental suffering is caused by the 'depersonalization' that occurs in most, if not all, big hospitals. It happens easily in a busy acute medical or surgical ward. As there is so much to do and so little time in which to do it, patients have to be regimented to enable the workload to be completed. The patient dying in the corner bed, possibly largely ignored, with no visits to the radiology or physiotherapy departments to provide an escape, easily feels he doesn't matter any more. Although it is his illness, he is not consulted about anything, his cooperation is not sought. Resentment at everything and with everybody begins to build up, symptoms tend to multiply and worsen-all because the patient is not being treated as a person. At St Christopher's we try to reverse the process. It begins at the moment a patient arrives at the door of the Hospice where he is welcomed by a warmed bed, rather than a cold impersonal trolley, and by the Matron or deputy who makes a point of greeting the patient by his name and shaking hands: 'Hullo, Mr. Smith, we are very pleased to see you: we hope you will be happy with us'. At first this might not seem at all important, but, personally, I think it is of great importance. It is the first step by which we hope to demonstrate to the patient that he matters to us and that he is going to be respected as a person and not just treated as a thing. The impact of the welcome can be quite extraordinary. On one occasion, a woman's symptoms of pain and general distress had virtually vanished by the time she reached the ward. Another time the wife of a patient said to the Matron, as her husband was being transported to the ward, 'It is marvellous to know that my husband is going to be treated as a person again'. In short, a simple act of courtesy can greatly reduce anxiety, go a long way to restore a person's self respect and, consequently, help considerably in the control of pain and other symptoms.

Patients in pain require analgesia. Most doctors would agree that simple analgesics should be tried in the first instance. The right dose of any analgesic is that which gives adequate relief for a reasonable period of time. Patients do not want to be constantly swallowing tablets or receiving injections. A 4 hour interval between doses should be regarded as the norm, though, on occasion, a shorter interval may be necessary. The abbreviation 'p.r.n.' has little or no place in the treatment of cancer pain: chronic pain, whatever the aetiology, requires regular preventive therapy. Once a drug has been shown to be ineffective the patient should be transferred to an alternative. Eventually the alternative will be a narcotic analgesic such as dipipanone, methadone or phenazocine.

When and if the pain becomes so severe that synthetic narcotic analgesics fail to provide adequate relief, one should not hesitate to turn to morphine or diamorphine. Many doctors fear both these preparations. I would suggest that in the situation we are discussing, this fear is entirely unjustified. Bogeys of addiction and escalating dosage often result in the statement, 'I think we ought to save the morphine until things get really bad'. Such statements to a relative can be guaranteed to do just that-intensify the pain in the patient-but still the morphine is not forthcoming. When other drugs have failed I would suggest a doctor is failing in his duty if he does not prescribe morphine or diamorphine.

At St Christopher's Hospice diamorphine is used 
in preference to morphine. Most patients are able to take it orally as a diamorphine-cocaine elixir.* They are maintained on a suitable dose given regularly every $4 \mathrm{hr}$ to prevent recurrence of pain. Some never need more than $2.5 \mathrm{mg}$ per dose whilst others receive $30 \mathrm{mg}$ or more. Furthermore, with the aid of a night sedative many patients do not require a 2 a.m. dose, though, if it is found to be necessary, the patient is wakened to have it rather than wake up later complaining of a recurrence of pain. Ultimately, most patients are transferred to parenterally administered diamorphine for the last 12 to $24 \mathrm{hr}$ on account of increasing difficulty with swallowing or coma; the dose given is half the previously satisfactory oral one. Almost all our patients receive a phenothiazine syrup with the diamorphine elixir, usually either prochlorperazine or chlorpromazine. Originally given to relieve co-existent nausea or vomiting, it is now also given to mask the bitter taste of diamorphine. It is possible, however, that the well-documented analgesic potentiation seen with many phenothiazine derivatives accounts, at least in part, for the relatively small doses of diamorphine that are the norm at St Christopher's Hospice.

Lung cancer can cause pain in many different parts of the body (Table 3 ). The pain of a bone metastasis

TABLE 3. Mechanism of pain in lung cancer

\footnotetext{
1. Extension of the primary neoplasm

2. Metastasis to bone

3. Raised intracranial pressure

4. Brachial plexus involvement

5. Hepatic involvement

6. Soft tissue metastasis
}

may be eased by the sensible use of analgesics, but, as pointed out earlier today, radiotherapy should always be considered in such cases. Headache due to raised intracranial pressure is best treated with a glucocorticosteroid with or without a simple analgesic. Most commonly we use dexamethasone $2-4 \mathrm{mg}$ up to 4 times a day, though sometimes we use the less potent prednisone. This regimen usually achieves pain relief without depression of the sensorium. By contrast, narcotic analgesics may well cause the situation to become worse by producing hypercapnia as a result of respiratory depression. Hypercapnia is always accompanied by an increase in intracranial pressure. Nevertheless, if simple analgesics and dexamethasone fail to provide relief one must be prepared to prescribe an adequate dose of a narcotic analgesic.

\footnotetext{
* Diamorphine $10,20,30$ or $40 \mathrm{mg}$; Cocaine $10 \mathrm{mg}$; Ethyl Alcohol 95\% B.P. $2.5 \mathrm{ml}$; Syrup $(66 \%$ Sucrose in water) $5 \mathrm{ml}$; Chloroform Water to $20 \mathrm{ml}$.
}

\section{Case history 2}

When investigated in June 1972 for giddiness and headache a 62-year-old builder was found to have carcinoma of the right main bronchus. In October 1972, the cerebral metastasis was irradiated in an attempt to control his symptoms. Subsequently, the primary growth was irradiated on account of severe chest pain. The latter course of radiotherapy was effective, the former was not. He still experienced severe, intermittent headache and told his relatives that he could not take much more: he was depressed by his failure to improve after treatment.

When admitted in December 1972, he could walk to the toilet, but spent most of the time in bed. He had no appetite and complained of mild recurrent haemoptysis. Apart from alopecia, examination was unremarkable. He was treated with prednisone $5 \mathrm{mg}$ t.d.s. and nitrazepam $10 \mathrm{mg}$ together with distalgesic tabs 2 at night. After 3 days he said he felt marvellous and had regained his appetite. He spent Christmas at home but on his return to hospital developed bronchitis. This was treated in the usual way with an antibiotic and bronchodilator. On 13 January, 1973 , he was discharged taking only prednisone $5 \mathrm{mg}$ t.d.s. Apart from a further attack of bronchitis he has subsequently remained well, being cared for by his somewhat over-protective wife.

An apical lung cancer (Pancoast tumour) is liable to invade not only adjacent bone but also the nerves of the brachial plexus. Whereas involvement of the first or second rib gives rise to local pain, involvement of the brachial plexus produces sensory or motor disturbances in the arm, and involvement of the sympathetic nerve chain results in an ipsilateral Horner's syndrome. The pain felt in the arm is incessant with periodic short sharp exacerbations. The sensible use of drugs will dull the pain considerably though the exacerbations usually defy pharmacological control. Two patients admitted recently with this syndrome both died within $48 \mathrm{hr}$. One deteriorated so rapidly that further assessment was impossible: the other was greatly helped by a combination of diamorphine $10 \mathrm{mg}$ and chlorpromazine $50 \mathrm{mg} 4$ hourly. After sleeping soundly the second night he woke up refreshed and relaxed, but died suddenly an hour or two later. Most patients with Pancoast's syndrome will, however, probably be referred at a somewhat earlier stage to a Pain Clinic where, if analgesics prove inadequate, a phenol nerve block or transcutaneous tractotomy may be recommended.

\section{Dyspnoea and cough}

These usually go together. As with pain, anxiety and fear greatly potentiate the distress that accompanies severe dyspnoea. For this reason both diazepam (Valium) and diamorphine have a place 
in the treatment of dyspnoea due to malignant disease. If the dyspnoea is associated with wheezing, an increase in the dose of a previously satisfactory bronchodilator may help considerably. The use of prednisone should be considered. Left ventricular failure is occasionally the cause of severe dyspnoea in these patients and should be treated with digoxin and diuretics. If simple linctuses fail to ease the cough, diamorphine or morphine should be used. On the other hand a purulent cough is often best treated with a broad spectrum antibiotic. Bromhexine (Bisolvon) can be a great help when a patient complains of difficulty in expectorating tenacious sputum.

\section{Case history 3}

In the autumn of 1971, Mr W.E., a 63-year-old schoolmaster and longstanding bronchitic, was found to have bronchial carcinoma affecting the right midzone. Despite radiotherapy he steadily deteriorated. When admitted on 10 March 1972 he was bedfast on account of extreme dyspnoea and also had moderately severe chest pain. Treatment included diamorphine (by mouth), prednisone, salbutamol, Bisolvon, Septrin and frusemide together with potassium supplements. He gradually improved on this regimen. The Bisolvon was stopped after 3 weeks and the diamorphine replaced by diazepam.

In May he was discharged, being fully mobile and able to enjoy life. The dose of prednisone had been reduced from the original $20 \mathrm{mg}$ t.d.s. to $10 \mathrm{mg}$ t.d.s. He was re-admitted 6 weeks later on account of increasing dyspnoea due to pneumonia; his antibiotic was changed to oxytetracycline and diamorphine reintroduced. After 3 weeks, in July 1972, he returned home still on diamorphine. In September he was re-admitted generally less well. He developed troublesome bone secondaries, spent more and more time in bed and began to have periods of confusion. He was, however, kept comfortable by the continued use of diamorphine. He died towards the end of October 1972.

\section{Anorexia and weight loss}

Both are common in terminal lung cancer patients. It is easier to correct anorexia than weight loss. Appetite often improves simply by admitting a patient to hospital-especially in the elderly or a person living alone. Some note improvement when symptoms such as nausea and pain are controlled. In others, where the anorexia is a manifestation of depression, treatment with, for example, amitriptyline causes a return of appetite. When all else fails it is worth trying prednisone $5-10 \mathrm{mg}$ t.d.s. for up to 2 weeks. If effective it can be continued, otherwise, it should be stopped.
Some patients receiving chlorpromazine or a glucocorticosteroid become hyperphagic. This is usually welcomed by the patient although, occasionally, modification of the patient's drug regimen is necessary. Weight loss in association with malignant disease is not solely the result of anorexia. Consequently, a return of appetite does not mean that weight loss will be corrected or even halted.

\section{Nausea and vomiting}

There was no significant difference between those patients who had received radiotherapy and those who had not in relation to nausea and vomiting. Both nausea and vomiting are usually relieved either by a phenothiazine, metoclopramide (Maxolon) or cyclizine.

\section{Haemoptysis}

When the haemoptysis is little more than bloodstained sputum, reassurance is usually all that is necessary. Frank haemoptysis can be controlled by prednisone or radiotherapy. Massive haemoptysis requires sedation with an intramuscular opiate and a nurse to hold the patient's hand. Very rarely, the massive haemoptysis is best described as 'catastrophic haemorrhage'. This results from erosion of the aortic arch or one of its main branches. There is no treatment: the patient dies immediately.

\section{Miscellaneous}

Hoarseness due to left recurrent laryngeal nerve palsy is untreatable but reassurance that the voice will not fade away entirely is usually welcomed by the patient. Superior vena caval obstruction, when it occurs, varies in intensity. It usually responds to treatment with a diuretic and prednisone. If these do not help radiotherapy should be considered. Fractured femurs and paraplegia occur from time to time. Provided the patient is reasonably well in himself, pinning of the femur should be considered. Incipient paraplegia due to vertebral erosion and collapse can often be arrested by irradiating the involved vertebral body.

Patients who have had thoracic surgery, i.e. lobectomy or pneumectomy, have a greater incidence of cerebral secondaries compared with those who have not. On the other hand, in patients who have not had surgery there is a greater incidence of haemoptysis, pain, anorexia and weight loss. The mode of death of those patients is summarized in Table 4. Massive haemoptysis and catastrophic haemorrhage are both rare.

\section{Conclusion}

Terminal care does not, however, stop at the drug trolley. Sir David Smithers recently said that no-one who hasn't time for chat knows anything about 
TABle 4. Mode of death in lung cancer

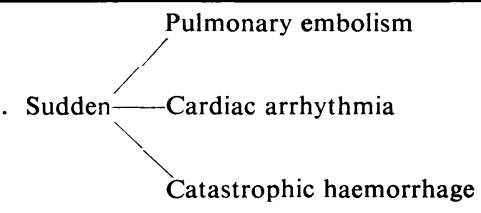

2. Rapid deterioration Pneumonia

3. Coma due to cerebral secondary

4. Gradual deterioration

terminal care (Smithers, 1973), and 'chat' in this context means patient chat while the doctor listens. Most patients want to talk about their illness, to express their fears and anxiety, to seek reassurance that 'it will not be too long', that they will not suffocate or choke to death. For example, Mr C.J.B. (Case History 1) when admitted said he would like to put his head into a gas chamber. This was understandable in view of his condition; alone and virtually bedfast for 6 weeks. A week later, though washed and shaved and symptomatically improved he still wanted to kill himself. After a further week I asked him again if he still wished to gas himself. He replied, 'I don't want to go home'. It then came out that he was living in a flat at the top of his son's house and the relationship between them was more than strained. On no account did he wish to go back there. He was relieved when I assured him that we would not expect him to go back. For the rest of his admission, he was fully active and enjoyed life in the hospice. He definitely no longer wished to die. On this occasion we failed to patch up the family quarrel. Sometimes, however, we are able to help by bringing the two sides together.

Terminal illness should not be regarded as an interlude or intrusion into life. It is part of life and can be a most positive experience-a time of increasing maturity and deepening relationships. It is our job as doctors to help it be so.

\section{Acknowledgments}

The author wishes to thank Dr Joan Haram for encouraging him to study patients with lung cancer apart from those with other forms of malignant disease, and also Miss Susan Cayre for preparing Table 1 . The author is in receipt of a research fellowship from the Sir Halley Stewart Trust.

\section{References}

Smithers, D. (1973) Care of Dying: Where to die. British Medical Journal, 1, 34.

Watson, L. (1966) Calcium Metabolism and Cancer. Australasian Annals of Medicine, 15, 359.

\title{
Discussion
}

\author{
Chairman: DR J. R. MikHAIL
}

(Central Middlesex Hospital)

DR SCADDING: In your patient who recurred after 11 years, was it the same histology or was it a new primary?

DR TwYCRoss (St Christopher's Hospice): I meant to say it could well have been a second primary. It is interesting to note that in those 2 years I was only able to put up 185 patients to take those symptoms from. There were eight other patients but they had to be excluded because they all had associated other primary carcinomas and I think that with a common disease such as lung cancer the possibility of a second lung cancer cannot be excluded.

Dr K. P. Ball (Central Middlesex Hospital): I was very interested to hear you comment about the importance of chat. I remember Dr Arthur Wilox, a physician that many of us will remember from the Middlesex, used to underline the importance of not hurrying past a dying patient's bed. It is very easy to do it on a busy round when one has other people to see for whom one could think you can do more. Another problem, of course, is that there are not enough St Christopher's Hospices about. Many of the kinds of things you have been recommending there are difficult to do in a general hospital. Obviously we ought to apply many more things in our own wards but we cannot do all of them.

DR TwYCRoss: I accept there are problems, and I hope I made it clear that I fully understood it, having worked in general hospitals for some time before I took up this research fellowship. I do not know what the answer is except to follow the advice of the physician you referred to, and that of Sir David Smithers. I think it must be difficult for someone who is under the care of a radiotherapist or a chest physician who has been working trying to cure them and then suddenly at some time you have got to face up to the reality that you have failed. Gloom and everything goes rapidly across, not only the medical team but the relatives and the patient. I don't know whether it is possible to get round this. We start afresh because we take them on and we have a fresh approach. We don't have a legacy of failure and we don't look at these patients as having incurable cancer. We tend to think of them as having diseases of pain, weakeness, anorexia, etc. We set out to treat those, and when you have clerked a patient we say 'Well, look. Your problem seems to me (1) you have got pain, (2) you 